TRABAJOS DE PREHISTORIA

66, N. ${ }^{\circ} 1$, enero-junio 2009, pp. 99-113, ISSN: 0082-5638

doi: $10.3989 /$ tp.2009.09014

\title{
NOTICIARIO
}

\section{Nuevas fechas radiométricas para la Prehistoria del noroeste de la Península Ibérica: la cueva de Valdavara (Becerreá, Lugo)}

\author{
New radiometric dates for the Prehistory of Northwestern Iberia: Valdavara Cave \\ (Becerreá, Lugo)
}

\author{
Manuel Vaquero Rodríguez (*) \\ Susana Alonso Fernández (*) \\ Carmelo Alonso Fernández (?) \\ Alicia Ameijenda Iglesias (***) \\ Hugues-Alexandre Blain (*) \\ Ramón Fábregas Valcarce $(* * *)$ \\ Gala Gómez Merino (**)
}

\section{RESUMEN}

El objetivo de este artículo es dar a conocer los primeros resultados de las excavaciones en la cueva de Valdavara (Becerreá, Lugo), prestando especial atención a las dataciones radiométricas que la sitúan entre los pocos yacimientos gallegos que han arrojado fechas correspondientes al Paleolítico Superior. La excavación realizada en el año 2007 permitió documentar dos localizaciones arqueológicas en el marco del mismo sistema cárstico (Valdavara 1 y Valdavara 2). El sondeo iniciado en Valdavara 1 ha permitido identificar hasta el momento dos conjuntos estratigráficos: un conjunto superior correspondiente a la Prehistoria reciente y un conjunto inferior (nivel 4) en el que se han obtenido las fechas del Paleolí-

(*) Área de Prehistoria. Universitat Rovira i Virgili. Pl. Imperial Tarraco, 1. 43005 Tarragona. Correos electrónicos:

manuel.vaquero@urv.cat, jlopez@prehistoria.urv.cat jordi.rosell@urv.cat

(**) Institut Català de Paleoecologia Humana i Evolució Social (IPHES). P1. Imperial Tarraco, 1. 43005 Tarragona. Correos electrónicos:

gala@prehistoria.urv.cat, mlozano@prehistoria.urv.es hablain@prehistoria.urv.cat, clorenzo@prehistoria.urv.cat

$(* * *)$ Grupo de Estudos para a Prehistoria do NW. Dpto de Historia I. Universidade de Santiago de Compostela. 15782 Santiago de Compostela. Correos electrónicos:

alicia.ameijenda@rai.usc.es, ramon.fabregas@usc.es alombera@prehistoria.urv.cat,mremedios.serna@usc.es carlos.rellan@usc.es.

Recibido: 9-VI-2008; aceptado: 11-XII-2008

\author{
Arturo de Lombera Hermida (**)/(***) \\ Juan M. López-García (*) \\ Carlos Lorenzo Merino (*) \\ Marina Lozano Ruiz (**) \\ Carlos Rodríguez Rellán (***) \\ Jordi Rosell i Ardèvol (*) \\ María R. Serna González (***)
}

tico Superior final. En Valdavara 2 se encontró un conjunto de restos humanos de la Edad del Bronce.

\begin{abstract}
The goal of this paper is to make known the first results of the excavations in Valdavara Cave (Becerreá, Lugo), paying special attention to the radiometric dating that places this cave among the few Galician sites with Upper Palaeolithic dates. During the excavation carried out in 2007, two archaeological locations were documented in the same karstic system (Valdavara 1 and Valdavara 2). The test pit initiated in Valdavara 1 allowed us to identify so far two stratigraphic units: an upper unit corresponding to Recent Prehistory and a lower unit (level 4) that yielded the Upper Palaeolithic dates. An assemblage of human remains from the Bronze Age was found in Valdavara 2.
\end{abstract}

Palabras clave: Paleolítico Superior; Prehistoria reciente; Fechas radiométricas; Galicia; Cueva de Valdavara.

Key words: Upper Palaeolithic; Recent Prehistory; Radiometric dating; Galicia; Valdavara Cave.

\section{INTRODUCCIÓN}

El objetivo de este artículo es presentar los resultados preliminares de las intervenciones ar- 
queológicas en la cueva de Valdavara, prestando especial atención a las primeras fechas de su secuencia estratigráfica, que se añaden al escaso registro radiométrico existente hasta el momento para el Paleolítico Superior gallego. Las excavaciones se iniciaron el año 2007, en el marco del proyecto de investigación Ocupaciones humanas durante el Pleistoceno en la cuenca media del Miño, desarrollado por la Universidad de Santiago de Compostela y la Universidad Rovira i Virgili. En este proyecto se enmarcan también las prospecciones en la depresión de Monforte de Lemos y las intervenciones en la Cova do Xato (Fábregas et al. 2007; Fábregas et al. 2008).

La cueva de Valdavara se encuentra situada en el Concello de Becerreá (Fig. 1), en el extremo oriental de la provincia de Lugo (UTM X: 5241085, Y: 474533126), a una altitud de unos 600 m s.n.m. Desde el punto de vista geológico, se localiza dentro del Dominio del Manto de Mondoñedo, una de las unidades en las que se subdivide la Zona Asturoccidental-leonesa, que a su vez forma parte del Macizo Ibérico. Esta zona está constituida por formaciones del Paleozoico Inferior, con un predominio de rocas arenosas y pizarrosas, si bien destaca la presencia de formaciones calcáreas. La cueva de Valdavara se abre en las calizas del Cámbrico Inferior-Medio (Calizas de Vegadeo), que afloran a lo largo de una amplia franja de trazado NNO-SSE. Se trata de una formación muy singular por su litología, constituida por dolomías y calizas. El contenido fosilífero de los niveles infra y suprayacentes sugiere su atribución al Cámbrico Inferior, aunque sus niveles más altos podrían alcanzar el Cámbrico Medio (Vera 2004).

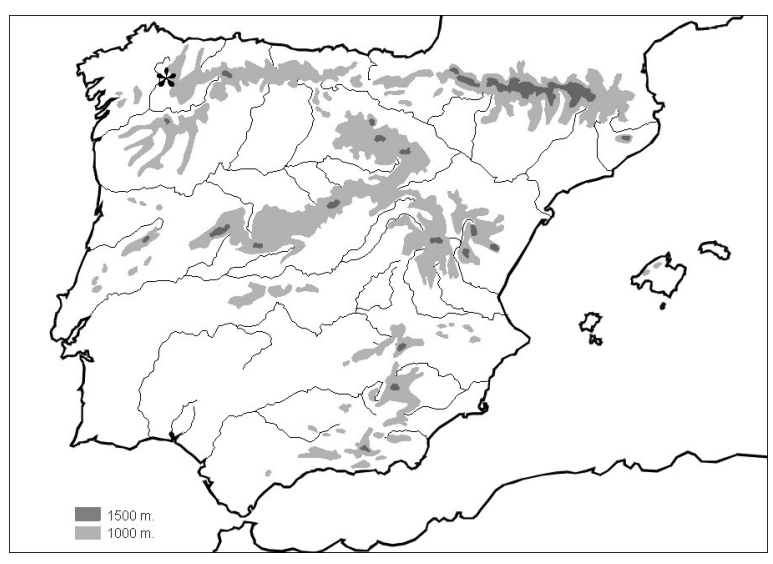

Fig. 1. Situación de la cueva de Valdavara.
Valdavara es una de las numerosas cuevas que forman parte de las formaciones cársticas existentes en el entorno del municipio de Becerreá (Lám. I). Alguna de estas cuevas era ya conocida por haber proporcionado algunos restos arqueológicos o paleontológicos aislados, como la Cueva de Furco o la Cova da Venta (Fernández Rodríguez 1993; Grandal 1991). Sin embargo, nunca hasta el momento habían sido objeto de un proyecto de investigación encaminado a establecer su relevancia arqueológica. La cueva se localiza en el margen derecho del río Narón, que recibe en esta zona el nombre de Cruzul, uno de los afluentes del Navia, a una altura de unos $120 \mathrm{~m}$ sobre el cauce actual. Se trata de una pequeña cavidad abierta hacia el noreste, con una entrada de apenas $1,2 \mathrm{~m}$ de anchura y 1,6 $\mathrm{m}$ de altura que da acceso a una sala de unos $5 \times 3 \mathrm{~m}$, al fondo de la cual se bifurcan dos galerías de escaso recorrido debido al relleno sedimentario que las va colmatando progresivamente.

La información sobre la existencia de un yacimiento arqueológico en Valdavara nos la proporcionaron vecinos de Becerreá. A finales de los años sesenta, un grupo de aficionados locales realizó un pequeño sondeo de unos $120 \mathrm{~cm}$ de profundidad cerca de la entrada de la cueva. Este sondeo proporcionó un conjunto de materiales que se ha conservado hasta el presente en una colección particular que tuvimos la oportunidad de examinar antes de iniciar los trabajos. Entre estos restos se incluían fragmentos óseos y cerámicos, así como artefactos líticos en sílex y cuarzo. También se constató la presencia de algunos restos humanos que sugería el uso de la cueva como lugar de enterramiento. Las características de estos materiales apuntaban la posibilidad de que en el conjunto estuviesen representados al menos dos períodos cronoculturales diferentes. Por un lado, los restos cerámicos indicaban la presencia de evidencias correspondientes a la Prehistoria reciente. Por otro lado, entre los restos líticos se detectó un lote de piezas en sílex que indicaba una fase ocupacional más antigua, probablemente de época pleistocénica. En concreto, se identificaron algunos buriles característicos del Paleolítico Superior final. No obstante, se desconocía si estos materiales procedían de unidades estratigráficas diferenciadas o si, por el contrario, se trataba de un único paquete sedimentario revuelto en el que se encontraban mezclados elementos de distintas cronologías. 


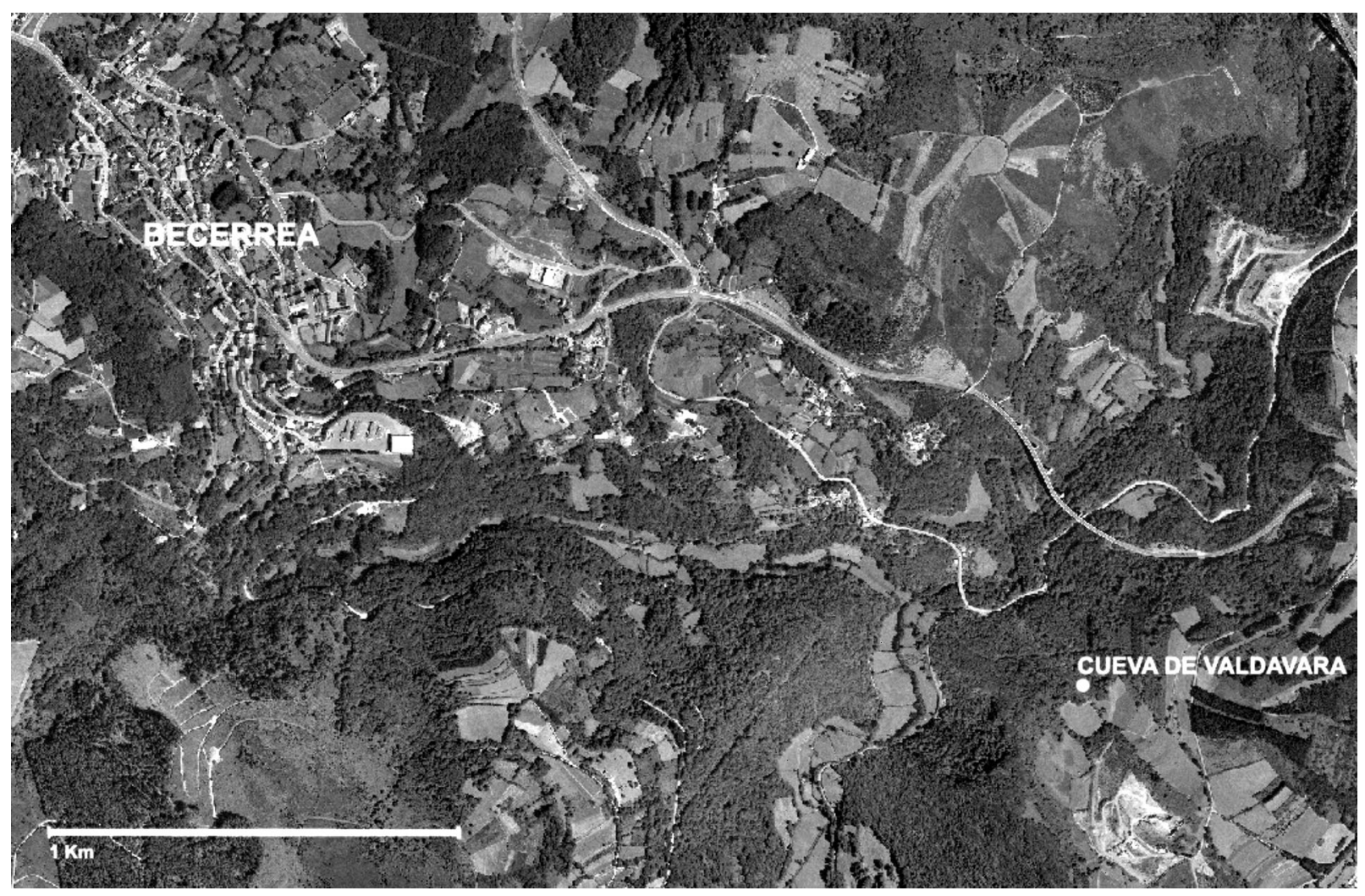

Lám. I. Localización de la cueva de Valdavara en el entorno del municipio de Becerreá.

Al realizar las primeras visitas al yacimiento pudimos comprobar que a escasos metros por debajo de dicha cavidad se abría, al pie de una pared rocosa, una pequeña fisura que parecía corresponder a una segunda entrada al sistema cárstico. El hallazgo de algunos restos óseos y líticos en superficie sugería la existencia de evidencias arqueológicas en el relleno de esta segunda localización, a la que decidimos denominar Valdavara 2, mientras que la cueva ya conocida fue designada como Valdavara 1 (Lám. II). La intervención realizada en julio de 2007 se planteó con la finalidad de alcanzar dos objetivos fundamentales:

a) Por un lado, documentar la secuencia estratigráfica de Valdavara $1 \mathrm{y}$, sobre todo, contrastar la presencia de niveles pleistocenos.

b) Por otro lado, comprobar la existencia de niveles arqueológicos en el depósito de Valdavara 2.

\section{Valdavara 1}

En Valdavara 1 se inició la excavación de un sondeo de $1,5 \times 1 \mathrm{~m}$ en el centro de la galería principal, a unos $2 \mathrm{~m}$ de la entrada (Lám. III). La localización del sondeo partía de la cata realizada en los años sesenta, que permitía disponer de una referencia estratigráfica. La extracción del sedi-

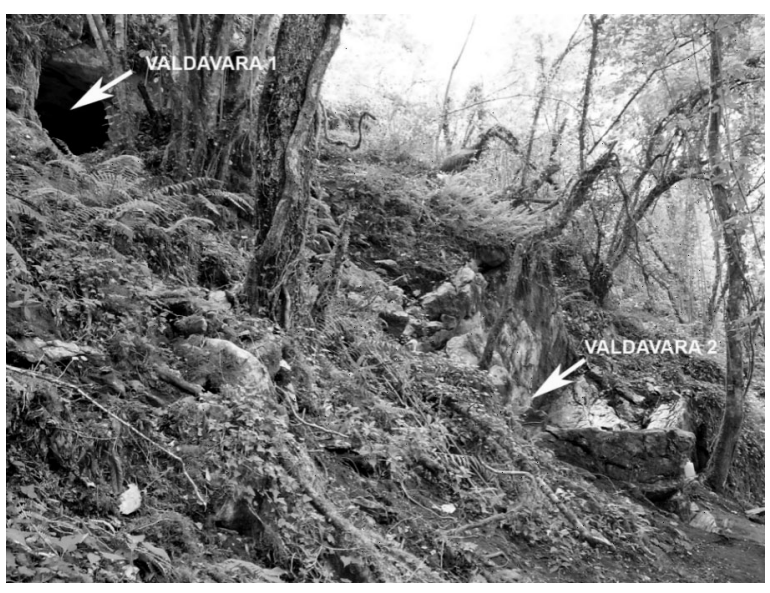

Lám. II. Vista general del yacimiento en la que se indica la localización de Valdavara 1 y Valdavara 2. Fotografía: S. Alonso. 
mento removido que rellenaba dicha cata, permitió recuperar un volumen considerable de restos arqueológicos, entre los cuales figuraban algunas piezas de industria lítica y ósea de aspecto paleolítico, así como fragmentos cerámicos y algunos restos humanos. Estas evidencias confirmaban los datos que se desprendían del conjunto exhumado en la intervención de los años sesenta. Una vez vaciado el sedimento removido, se pudo observar una secuencia de unos $120 \mathrm{~cm}$ de potencia, en la que se podían diferenciar dos conjuntos estratigráficos bien definidos, lo cual fue confirmado posteriormente por el sondeo. En dicho sondeo se han documentado hasta el momento cuatro unidades estratigráficas, si bien no se ha alcanzado todavía la base de la secuencia, por lo que ésta habrá de ser completada en campañas posteriores. Dejando a un lado el nivel 1, formado por

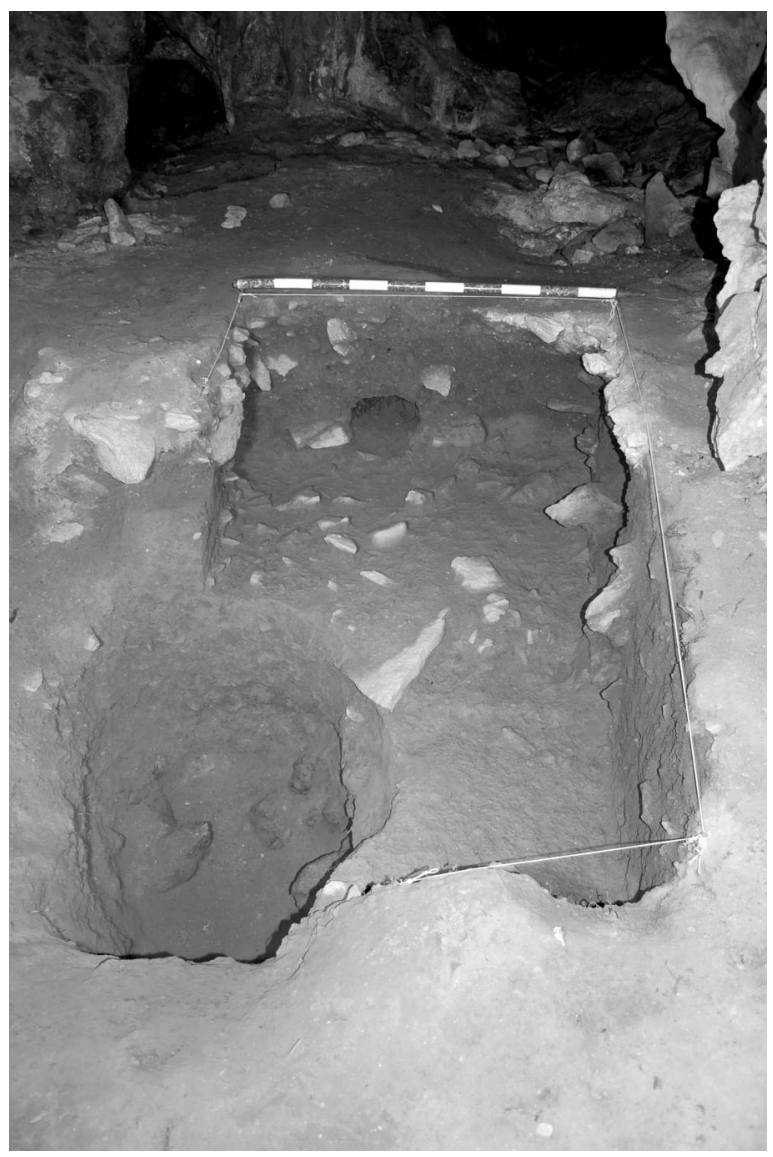

Lám. III. Imagen del sondeo practicado en Valdavara 1. En la parte inferior izquierda se puede observar la intervención realizada en los años sesenta. Fotografía: S. Alonso. una fina capa de tierra removida superficial, el resto de los niveles se estructura de la siguiente manera:

- Conjunto superior. Formado por los niveles 2 y 3 , es una capa de unos $40 \mathrm{~cm}$ de potencia de limos de color oscuro, con una coloración ligeramente más clara del nivel 3.

- Conjunto inferior. Constituido por el nivel 4, apenas se habían excavado unos 10-15 cm de espesor al finalizar la campaña. Formado por limos de color anaranjado, presenta un contacto erosivo muy neto con respecto al conjunto superior.

Esta clara distinción estratigráfica se vio corroborada desde el punto de vista arqueológico, ya que ambos conjuntos proporcionaron asociaciones de restos bien diferenciadas, tanto cultural como cronológicamente. El conjunto superior se caracteriza sobre todo por la presencia de fragmentos cerámicos y de una serie de restos humanos que apuntan hacia la utilización de la cavidad con finalidades funerarias. También se recuperaron varios fragmentos de Dentalium (Lám. IV). Estas evidencias, junto con las dataciones radiométricas que comentaremos más abajo, permiten adscribir este conjunto a la Prehistoria Reciente. En cambio, el conjunto inferior contempla la desaparición de la cerámica y la aparición de una serie lítica formada principalmente por artefactos en sílex. Como veremos, las fechas disponibles hasta el momento sugieren la atribución de este conjunto al Paleolítico Superior final. A continuación presentaremos el registro arqueológico

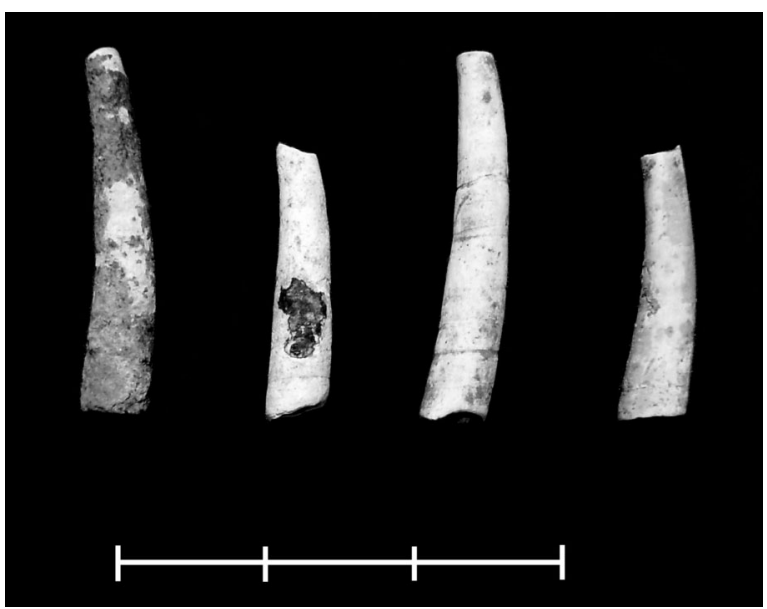

Lám. IV. Restos de Dentalium encontrados en el conjunto estratigráfico superior de Valdavara 1. Fotografía: S. Alonso. 
de ambos conjuntos, si bien hay que remarcar que los restos son todavía muy reducidos y hay numerosos aspectos que sólo podrán ser abordados a medida que se incremente la cantidad de material.

\section{Cerámica}

En lo que respecta al conjunto cerámico de los niveles 2 y 3, la mayoría de los fragmentos son amorfos, si bien se han documentado algunos bordes ( $7 \%)$, bases y fragmentos de cuello $(2 \%)$. A pesar de esta gran fragmentación, parecen dominar los tipos de tendencia ovoide o globular y los vasos de paredes rectas, con fondos planos y de talón. Las características de los bordes y paredes indican que pertenecen en general a recipientes de tamaño medio con superficies exteriores alisadas mates e interiores negras y con un espatulado. Las decoraciones son muy escasas, dominando las incisas sobre los cordones. Las primeras parecen corresponder a un mismo recipiente con perfil en S (Fig. 2: 2, 4 y 6). La decoración consiste en grupos de líneas paralelas y horizontales separadas por bandas lisas.

No hay demasiados elementos que permitan acercarse a la cronología de estas ocupaciones. A un ambiente genérico de Neolítico Final se vincularían los fragmentos del vaso con bandas de incisiones horizontales paralelas; piezas con decoración del mismo tipo aparecen ocasionalmente en contextos megalíticos, como en la mámoa 12 de Monte de San Miguel (Agolada, Pontevedra) (Fábregas et al. 2005). Lo mismo podría decirse de un gran fragmento de vaso de casquete esférico con $26 \mathrm{~cm}$ de diámetro de boca y engobe rojo bruñido, para el que tenemos un paralelo en contexto megalítico, en el sepulcro de corredor de A Cova da Moura (Noia, Coruña), donde se mencionan tres fragmentos con una aguada o engobe de estas características, que probablemente no correspondan a la primera utilización de la estructura funeraria y están en la misma tradición de pintadas y aguadas a la almagra de Millares y del Calcolítico occidental (Fábregas y Fuente 1988: 230 y Fig. 1.18). La investigación en poblados de Neolítico Final y Calcolítico de la Meseta Norte y también en algún sepulcro megalítico ha proporcionado cerámicas con decoración pintada, pero aquí domina la pintura negra y con ella se configuran motivos geométricos (Fabián 2006: 399-400). Estos mismos rasgos se aprecian en sendos fragmentos del poblado de Monte dos Remedios (Moaña, Pontevedra), con una atestiguada ocupación en el Neolítico Final y Calcolítico (Fábregas et al. 2007: 92).

No resulta fácil establecer una distinción temporal en un conjunto con alta fragmentación de los vasos (menos de $5 \mathrm{~cm}$ en su mayoría), que hace difícil su caracterización formal. Sin embargo cabe señalar la presencia, en alguna pieza, de determinados rasgos, como los fondos planos o con talón ligeramente reforzado, que podrían pertenecer a vasos de tipo ovoide y tal vez encajen mejor en un contexto de la Edad del Bronce. Desafortunadamente las características en la formación del registro (depósitos alterados por remociones relacionadas con los enterramientos secundarios y presencia de madrigueras) no permiten diferenciar por ahora esa diacronía entre los niveles holocenos.
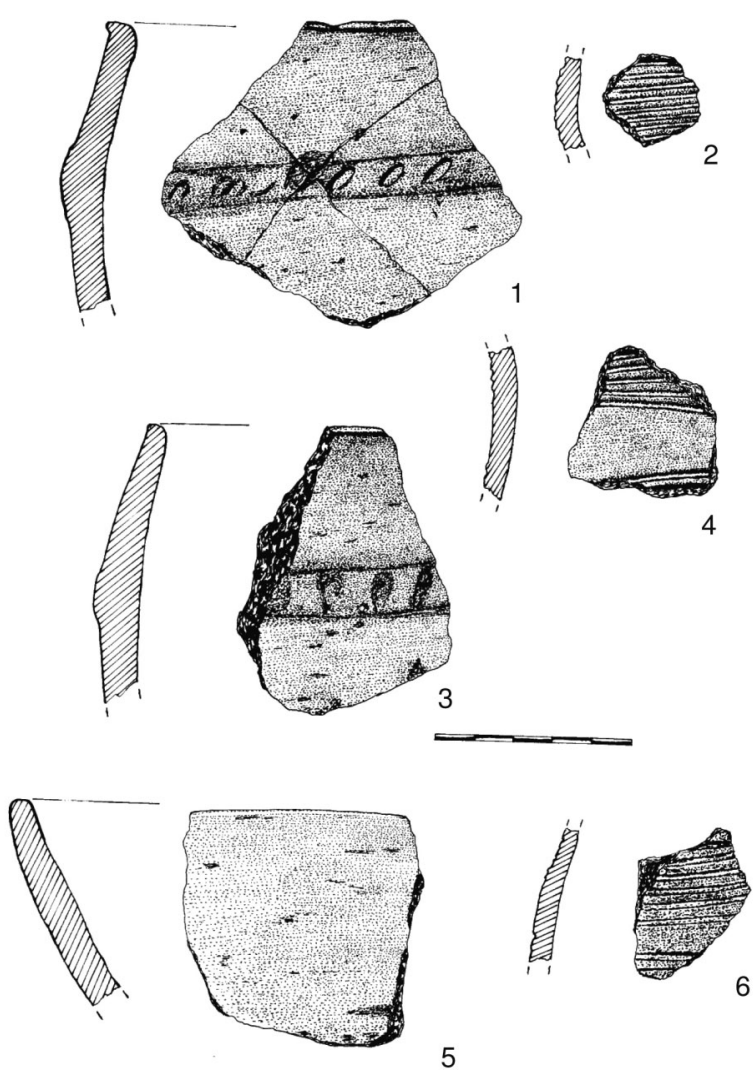

Fig. 2. Fragmentos cerámicos documentados en el conjunto superior de Valdavara 1. Dibujo: M. R. Serna. 


\begin{tabular}{|l|c|c|c|c|}
\hline & Nivel 4 & Nivel 3 & Nivel 2 & Total \\
\hline Caliza & & & 1 & 1 \\
Cuarzo & 12 & 6 & 17 & 35 \\
Ślex & 30 & 6 & 3 & 39 \\
Total & 42 & 12 & 21 & 75 \\
\hline
\end{tabular}

Tab. 1. Distribución por materias primas y niveles arqueológicos de los restos líticos coordenados en el sondeo de Valdavara 1.

\section{Industria lítica}

La industria lítica se caracteriza por un cambio marcado en las materias primas entre los conjuntos superior e inferior. Si bien el número de efectivos con los que contamos es todavía muy reducido (Tab. 1), los niveles 2 y 3 se caracterizan por un claro predominio del cuarzo, que representa casi el $70 \%$ de los restos líticos coordenados, frente al sílex. En cambio, en el nivel 4 estos porcentajes se invierten, pasando el sílex a ser la ma-
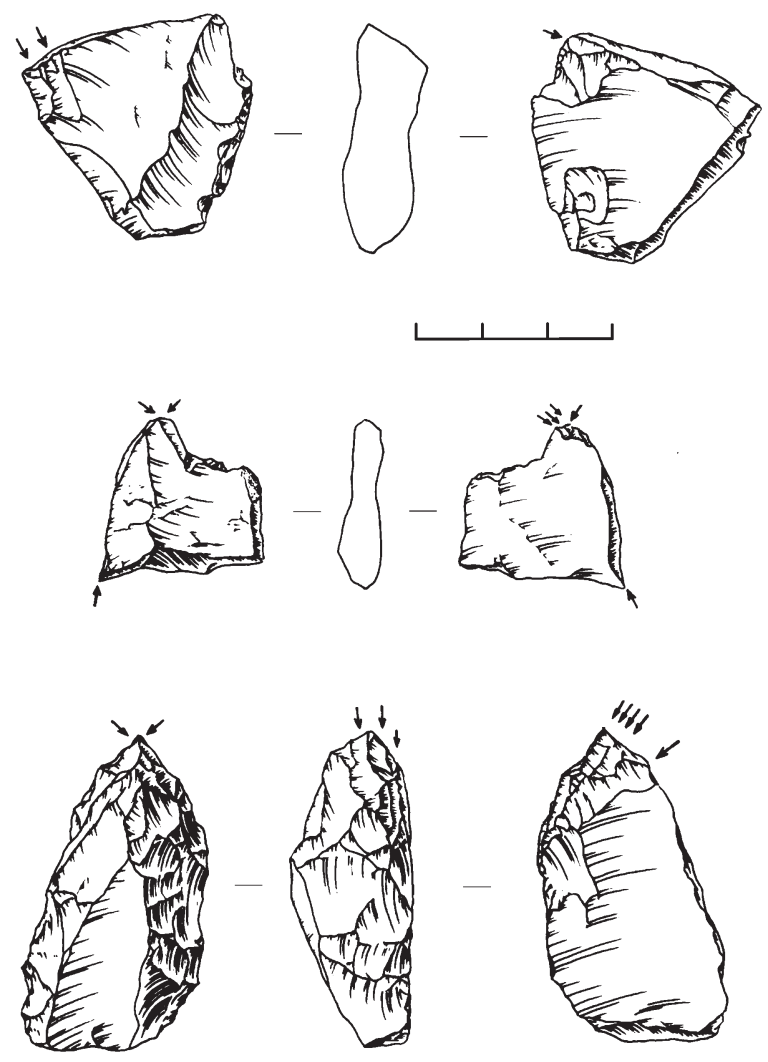

Fig. 3. Buriles sin contexto estratigráfico procedentes de Valdavara 1. Dibujo: S. Alonso. teria prima más representada, con más del $70 \%$ de los artefactos coordenados.

Más allá de esta diferenciación a nivel litológico, no han aparecido elementos realmente diagnósticos que permitan precisar el contexto cultural al que corresponden ambos conjuntos. Destaca la presencia bien contrastada de la talla laminar en el nivel 4 y la ausencia de datos que sugieran una selección volumétrica del material. Por el momento no se ha recuperado ningún artefacto retocado en el sondeo. Todos los artefactos retocados del conjunto procedente de la limpieza de la cata de los años sesenta, así como del recuperado durante dicha intervención, corresponden a buriles (Fig. 3). El sedimento adherido a alguna de estas piezas indica su procedencia del conjunto inferior de la secuencia estratigráfi$\mathrm{ca}$, al igual que algunos ejemplares de industria ósea encontrados también en el sedimento removido. Entre estos últimos destaca un fragmento de azagaya en asta de ciervo de unos $5 \mathrm{~cm}$ de longitud (Lám. V).

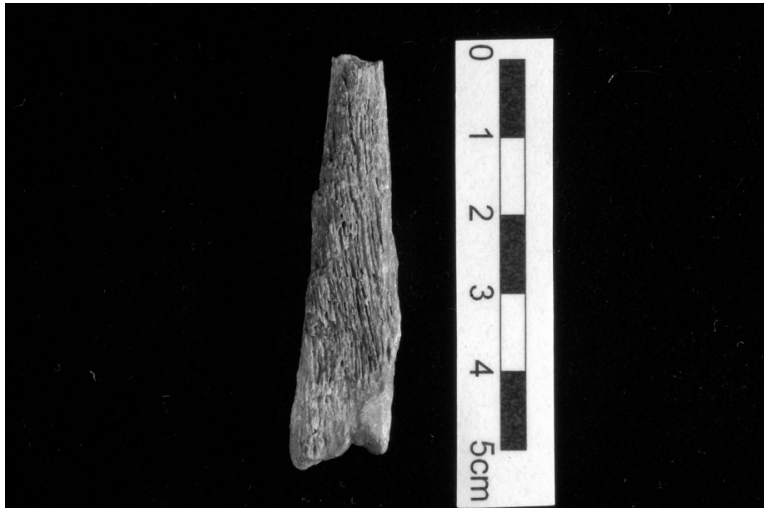

Lám. V. Fragmento de azagaya recuperado en los sedimentos removidos que rellenaban la intervención realizada en los años sesenta en Valdavara 1. Fotografía: Gerard Campeny. 


\section{Fauna}

El escaso número de restos óseos recuperado en el sondeo sólo permite realizar de momento una valoración preliminar de los conjuntos faunísticos. En el nivel 2 los animales de talla pequeña y muy pequeña son los más abundantes, aunque también se ha identificado un resto perteneciente a un animal de talla mediana. Destaca el dominio de los ovicaprinos, si bien los restos no permiten dilucidar si se trata de ovejas o cabras. También es muy significativa la presencia de pequeños carnívoros. En el nivel 3 no se encuentran taxones domésticos, destacando dos restos de cérvidos y un metacarpo de Vulpes vulpes. Los animales de talla pequeña también dominan el conjunto faunístico del nivel 4. Es de destacar la presencia del lobo, representada por una tercera falange, por ser éste un importante predador y acumulador de restos en cavidades. El rebeco también está documentado en el conjunto por un fragmento de ulna. Sólo existe en el conjunto un resto perteneciente a un animal de talla mediana. Las deposiciones de óxidos de manganeso están presentes en mayor o menor medida en todos los restos, indicando un ambiente húmedo y probablemente encharcado.

Por otra parte, Valdavara 1 ha proporcionado un abundante registro de microvertebrados. Los taxones identificados en los niveles 2 y 4 se incluyen en la tabla 2. Destaca la amplia diversidad de especies (31 taxones) tanto de micromamíferos (6 insectívoros, 1 quiróptero y 13 roedores), como de herpetofauna (2 urodelos, 3 anuros y 7

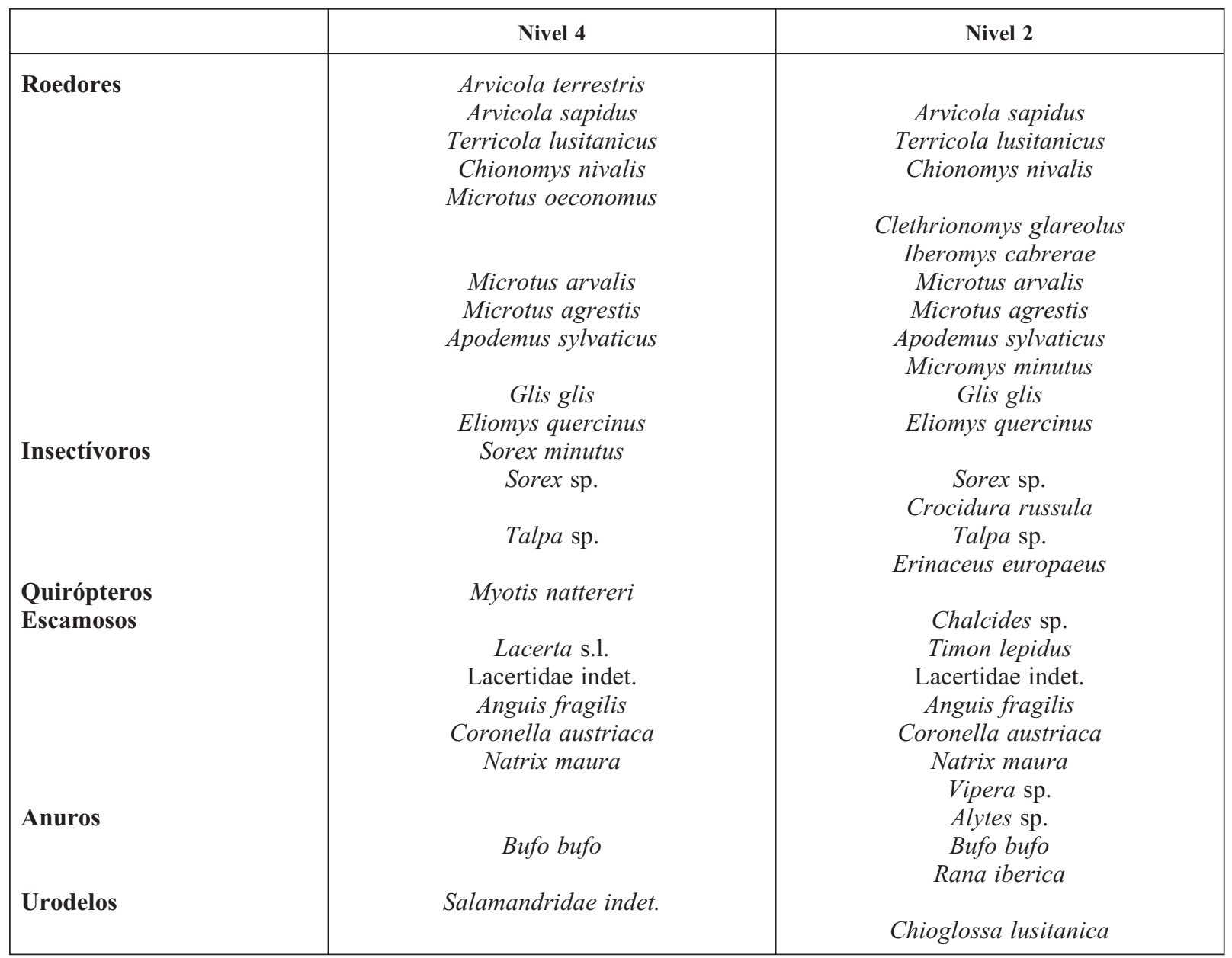

Tab. 2. Especies de microfauna documentadas en los niveles 2 y 4 de Valdavara 1. 
escamosos). Son destacables las diferencias entre las asociaciones de ambos niveles. La abundancia de Chionomys nivalis (topillo nival), junto a la presencia de Microtus oeconomus (topillo nórdico) y Sorex minutus (musaraña enana) en el nivel 4 define una asociación de pequeños mamíferos característica de la segunda parte del Pleistoceno Superior del norte peninsular, tal como se ha constatado en otros yacimientos como Las Caldas (Laplana et al. 2006), El Juyo (Pokines 1998), El Mirón (Cuenca-Bescós et al. 2009) o Amalda (Peman 1990). Por otro lado, la presencia de Clethrionomys glareolus (topillo rojo), $\mathrm{Mi}$ cromys minutus (ratón espiguero) y Crocidura russula (musaraña común) tan sólo en el nivel 2 es coherente con otras asociaciones de micromamíferos holocenas del Norte Peninsular, como las de El Mirón (Cuenca-Bescós et al. 2009) o Amalda (Peman 1990).

En ambos niveles, la asociación de pequeños vertebrados denota un ambiente muy húmedo con la presencia de $M$. oeconomus, $S$. minutus y los urodelos en general y, además, un paisaje alternante entre praderas húmedas y bosques de tipo atlántico. Por otra parte, las especies relacionadas con espacios forestales, como Apodemus sylvaticus (ratón de bosque), Glis glis (lirón gris) y en menor medida Anguis fragilis (lución), se encuentran mejor representadas en el nivel 2 que en el nivel 4. Las especies Arvicola sapidus (rata de agua), A. terrestris (rata topera), Natrix maura (culebra viperina) y Rana iberica (rana patilarga) indicarían en ambos niveles la existencia de cursos de agua estables próximos a la cavidad, con riberas de suelos sueltos favorables para especies cavadoras como Alytes sp. (sapo partero), A. fragilis, Chalcides sp. (seps) y Talpa sp. (topo).

Desde un punto de vista climático la presencia en el nivel 4 de varias especies relacionadas con un clima atlántico, como M. oeconomus, S. minutus y $C$. nivalis, sugiere un clima más fresco que el nivel 2, donde aparece $C$. russula, Iberomys cabrerae (topillo de cabrera) y Chalcides sp., que son actualmente especies de clima más mediterráneo. Esto concordaría con la presencia de Myotis nattereri (murciélago ratonero gris), especie altamente ligada a medios forestales, la cual habría utilizado la cueva como refugio durante una fase fría, como se ha observado en el caso de otros murciélagos con características ecológicas similares (Sevilla 1988).

\section{Restos humanos}

En Valdavara 1 se han recuperado hasta el momento 9 restos humanos, 3 de ellos en los sedimentos removidos y los 6 restantes en el nivel 2 . Destaca el hecho de que las únicas regiones anatómicas representadas son dientes $(\mathrm{n}=5)$ y falanges $(n=4)$ : 1 incisivo, 1 canino, 1 germen de premolar, 1 premolar, 1 primer molar deciduo, 1 falange proximal y 3 falanges intermedias. Aunque el número de restos humanos es reducido, los 5 dientes permiten reconocer, al menos, a 3 individuos:

- El primer individuo ha sido identificado a partir de un germen de $\mathrm{P}_{3}$ dcho. Esta pieza ha completado el desarrollo de la corona y ha iniciado levemente la formación de la raíz. Según Smith (1991), este individuo tendría una edad de muerte aproximada de 6-7 años.

- El segundo individuo se reconoce por un primer molar deciduo inferior derecho $\left(\mathrm{dm}_{1}\right.$ dcho). Este diente tiene las raíces en proceso de reabsorción y su corona un grado de desgaste con exposición de la dentina en la cresta lingual. La edad de muerte de este individuo se situaría en torno a los $9 \pm 1$ años (Ubelaker 1989).

- El tercer individuo corresponde a un adulto, identificado por el incisivo, canino y premolar permanentes con las raíces completas. El grado de desgaste muy marcado expone la dentina en los tres dientes.

Todas las falanges han completado el desarrollo y por lo tanto corresponden a un individuo adulto. Todas ellas son compatibles en tamaño y desarrollo y pueden asignarse al tercero de los individuos identificado a partir de los dientes.

\section{Valdavara 2}

Las intervenciones en Valdavara 2 se iniciaron levantando el nivel de tierra vegetal (nivel 1) que recubría todo el talud que se extiende al pie de la pared rocosa en la que se abre la cavidad. En esta capa se recuperaron algunos restos arqueológicos aislados, básicamente restos óseos y artefactos en cuarzo. Por debajo apareció una acumulación de grandes bloques de caliza fosilizada por un nivel de limos de color rojizo (nivel 2). Al llegar a este nivel, se focalizó la intervención en una superficie de unos $4 \mathrm{~m}^{2}$ situada inmediatamente al pie de la pared. A continuación se documentó un nivel de coloración similar al del nivel 2 pero con una 
mayor presencia de gravas, al que se denominó nivel 3. Lo más destacable de este nivel fue el hallazgo de un conjunto de restos humanos, en el que se incluyen elementos tanto del esqueleto craneal como del postcraneal.

A diferencia de Valdavara 1, el depósito estratigráfico de Valdavara 2 se caracterizó por la escasez de testimonios de cultura material. La excavación del nivel 2 proporcionó un conjunto formado básicamente por restos de fauna, con apenas evidencias claras de presencia humana. Lo mismo puede decirse del nivel 3, en el que, además de los restos humanos, se recuperó un conjunto faunístico relativamente abundante, cuyas características se comentarán a continuación.

\section{Fauna}

En el nivel 2 se ha recuperado un número muy escaso de evidencias faunísticas, de las cuales sólo han podido identificarse un resto de ovicaprino, no lo suficientemente diagnóstico como para atribuirlo a un género concreto (Capra u Ovis), y cuatro restos de lepóridos. El nivel 3 es el que ha proporcionado un mayor contingente de restos faunísticos, 49 de los cuales han sido identificados a nivel anatómico y taxonómico. Entre los taxones identificados se da la presencia de un gran bóvido atribuible al género Bos, del ciervo común, de un caprino indeterminado, de carnívoros de pequeño tamaño y de la familia de los lepóridos. Los animales que presentan más efectivos son los rebecos, seguidos a una cierta distancia por los lepóridos. Entre los restos de rebeco se observa un predominio de los huesos pertenecientes a las extremidades, sobre todo a las anteriores.

Bastantes restos muestran indicios de mordiscos o fracturas. La variabilidad métrica y morfológica de estas trazas, que van desde los chupeteos de los ápices distales de los huesos a las fracturas crenuladas y la presencia de improntas, indica que pertenecen a un carnívoro mediano y fracturador cuyo espectro de presas se caracteriza por el predominio de los animales de talla pequeña. Con todos estos elementos, es posible pensar que se trata de lobos. Se ha identificado deposiciones de manganeso en más de la mitad de restos, lo que indica que el medio de formación del conjunto presentaba una humedad acentuada. Ocasionalmente, los manganesos se asocian a

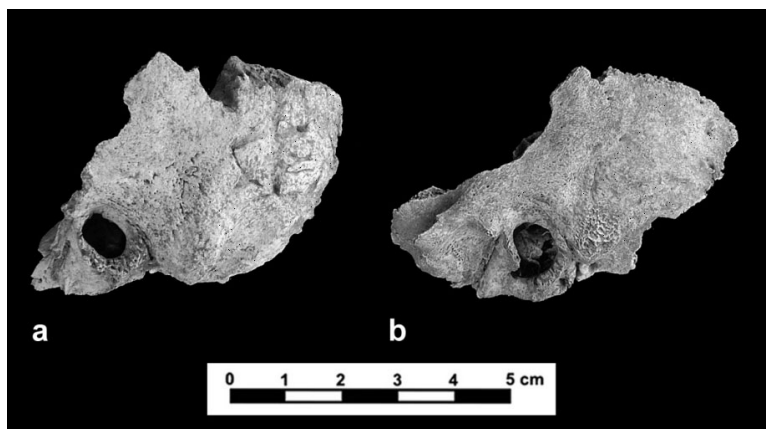

Lám. VI. Vista lateral de los temporales izquierdos de los dos individuos infantiles identificados en Valdavara 2: a) Individuo 1, con una edad de muerte de 2,5-3 años; b) Individuo 2, con una edad de muerte de 6-9 meses. Fotografía: Gerard Campeny.

concreciones. También se ha identificado, en valores muy bajos, la presencia de vermiculaciones de raíces antiguas y de corrosiones químicas.

\section{Restos humanos}

En Valdavara 2 se han recuperado 55 fósiles humanos. Todos proceden del nivel 3 y corresponden a dos individuos infantiles. El primero fue identificado en el yacimiento a partir del hallazgo de un neurocráneo bastante completo y otros huesos del postcráneo dispersos alrededor del cráneo que no estaban en conexión anatómica (Lám. VI). La revisión de los restos alrededor del cráneo ha permitido identificar un segundo individuo de una edad de muerte algo menor.

El individuo 1 tiene una edad de muerte de alrededor de 2,5-3 años, según se desprende del estado de osificación del hueso timpánico del temporal donde el tubérculo anterior y el posterior han completado su fusión y el anillo timpánico está completo (Krogman e Isscan 1986). También pueden asignarse a este individuo cuatro dientes deciduos aislados: incisivo central inferior izquierdo, incisivo lateral superior izquierdo, canino inferior derecho y canino superior izquierdo. Los dos incisivos han completado el desarrollo de la raíz mientras que a ambos caninos les faltan los ápices por lo que corresponden a un individuo con una edad de muerte cercana a los 3 años (Scheuer y Black 2000).

El individuo 2 tiene una edad de muerte inferior al anterior, entre 6 y 9 meses. Ha sido identificado gracias a un temporal izquierdo que presenta un estado de desarrollo del hueso timpánico donde el tubérculo posterior y el anterior 


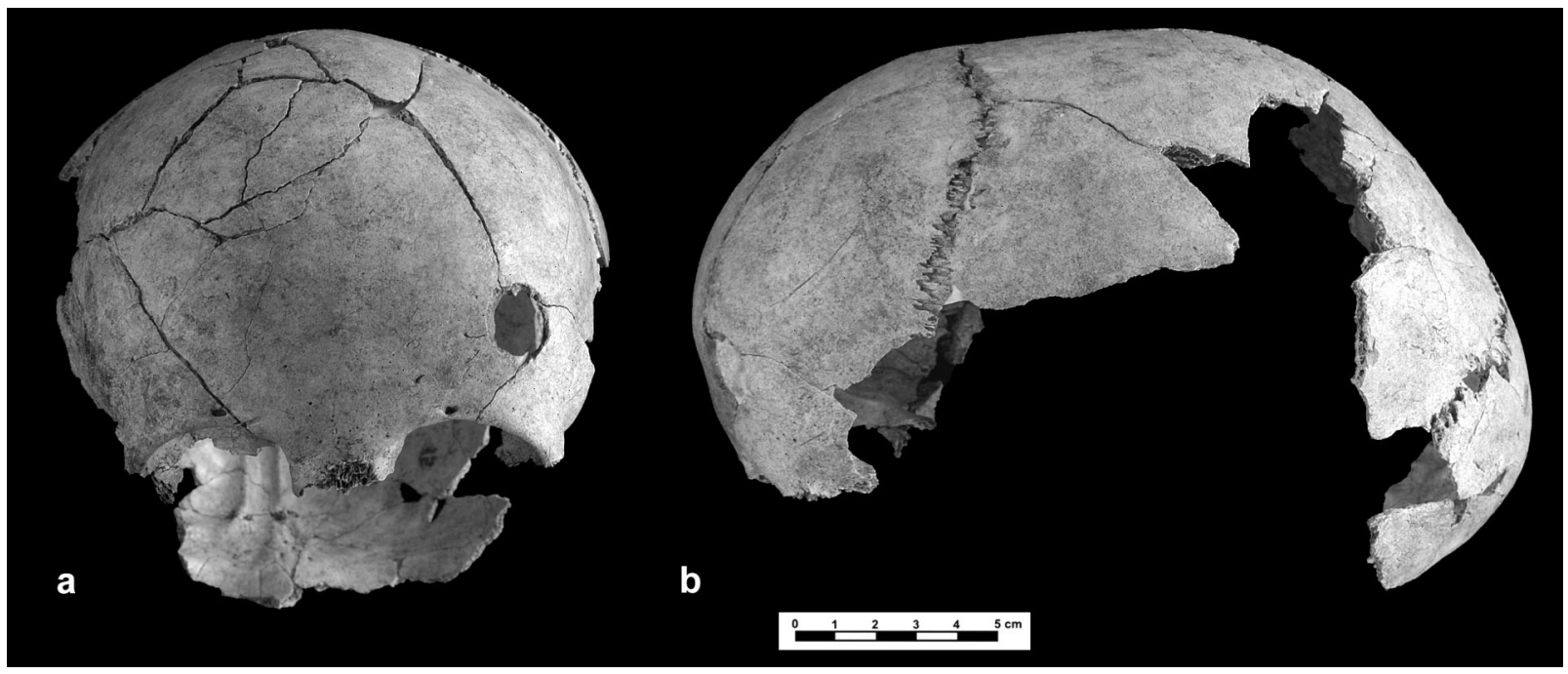

Lám. VII. Vista anterior (a) y vistal lateral izquierda (b) del cráneo infantil del individuo 1 de Valdavara 2. Fotografía: Gerard Campeny.

no están fusionados (Lám. VII). Además, dos dientes aislados podrían corresponder a este individuo debido a que el estado de desarrollo es compatible: primer molar deciduo superior derecho y germen del segundo molar deciduo superior derecho.

En cuanto a los huesos del esqueleto postcraneal, el hecho de tratarse de dos individuos muy jóvenes dificulta la determinación de la edad. No obstante, podemos proponer una asignación de algunos de los restos humanos postcraneales a partir de su tamaño y desarrollo. Al individuo 1 hemos adscrito 1 fragmento de escápula, 1 fragmento de húmero, 3 fragmentos de peroné, 1 fragmento de tibia, 1 fragmento de mandíbula y 2 fragmentos de radio. Al individuo 2 podemos asignar 1 fragmento de escápula, 1 fragmento de húmero y 1 de peroné.

\section{FECHAS RADIOMÉTRICAS}

Se han realizado hasta el momento cinco dataciones por ${ }^{14} \mathrm{C}$ (AMS) en los laboratorios Beta Analytic. Estas fechas se han obtenido a partir de restos óseos procedentes tanto de Valdavara 1 como de Valdavara 2 (Tab. 3). La datación del nivel 2 de Valdavara 1 ha sido realizada a partir de una falange humana perteneciente a un individuo adulto, mientras que las muestras del nivel 4 corresponden a dos fragmentos de diáfisis para los que no es posible realizar una atribución taxonómica. Las dos dataciones de Valdavara 2 han sido realizadas sobre huesos humanos. En Valdavara 1 los resultados confirman el salto temporal entre los conjuntos superior e inferior de la secuencia estratigráfica. Mientras que se comprueba la atribución del conjunto superior a la Prehistoria re-

\begin{tabular}{|c|c|c|c|c|c|c|}
\hline Loc. & Nivel & Material & Ref. lab. & $\begin{array}{c}\text { Años } \\
\text { radiocarbónicos }\end{array}$ & Años cal. BP & Años cal. AC \\
\hline Val-1 & 2 & Hueso humano & Beta-235727 & $4490 \pm 40$ & $5340-4980$ & $3390-3030$ \\
Val-1 & 4 & Hueso & Beta-235728 & $13770 \pm 70$ & $17080-16880$ & $15130-14930$ \\
Val-1 & 4 & Hueso & Beta-235726 & $14630 \pm 70$ & $17890-17730$ & $15940-15780$ \\
Val-2 & 3 & Hueso humano & Beta-235729 & $3270 \pm 40$ & $3600-3400$ & $1650-1450$ \\
Val-2 & 3 & Hueso humano & Beta-235730 & $3250 \pm 40$ & $3600-3360$ & $1650-1410$ \\
\hline
\end{tabular}

Tab. 3. Fechas radiométricas. La calibración a 2 sigmas $(\mathrm{p}=95 \%)$ ha sido realizada utilizando la curva de calibración CalPal-2007-Hulu del software CalPal (Weninger y Jöris 2004).

T. P., 66, N. ${ }^{0} 1$, enero-junio 2009, pp. 99-113, ISSN: 0082-5638 doi: $10.3989 /$ tp.2009.09014 
ciente, el conjunto inferior se encuadra claramente dentro del Pleistoceno Superior final, con fechas que nos sitúan, como comentaremos a continuación, en el contexto del Magdaleniense Inferior-Medio.

\section{CONCLUSIONES}

Los resultados de las excavaciones arqueológicas en la cueva de Valdavara son aún preliminares, dado el carácter limitado de la intervención efectuada hasta el momento y el escaso registro del que disponemos. No obstante, entre los datos obtenidos a raíz de esta primera campaña de excavación vale la pena detenerse en dos aspectos que revisten un singular interés: por un lado, una aproximación al comportamiento funerario durante la Prehistoria reciente a partir del registro documentado en el conjunto superior de Valdavara 1 y en Valdavara 2; por otro lado, la confirmación de la existencia en Valdavara 1 de niveles atribuibles al Paleolítico Superior $\mathrm{y}$, concretamente, al Magdaleniense Inferior-Medio.

Tras la llamativa presencia en el paisaje que suponen los túmulos megalíticos durante el V y IV milenios, las fórmulas de enterramiento se tornan algo más crípticas en el noroeste desde inicios del III milenio AC, una vez que se produce la clausura de los sepulcros de corredor. Bien es cierto que tenemos numerosos indicios de la reutilización de los túmulos por parte de gentes campaniformes, pero ésta se produce soslayando la norma funeraria precedente. Por otra parte, todavía carecemos de una base documental suficiente para analizar e interpretar la aparente variabilidad que tiene lugar en el ámbito sepulcral entre mediados del III milenio AC y los finales del II. Efectivamente, en este amplio marco temporal tenemos indicios del levantamiento de nuevos túmulos, la reutilización de los megalíticos y el empleo de cistas y de fosas excavadas en el suelo (una síntesis para la Edad del Bronce en Bettencourt e.p.).

Si volvemos nuestra atención a las evidencias de tipo funerario en grutas, la parquedad de datos es aún más notoria, pues a la escasez de formaciones cársticas en nuestra región se une su restricción a un área muy poco explorada arqueológicamente como es la de las montañas orientales. Así, las referencias que tenemos son antiguas y poco fiables en la mayoría de los casos, si bien los materiales recuperados sugieren enterramientos entre el Calcolítico y el Bronce Medio, según los casos (Bettencourt e.p.; Fernández et al. 1995; Vázquez 1988). Un conocimiento más detallado nos lo da la cueva de Pala da Vella (Rubiá, Ourense), en la que los sondeos efectuados definieron un nivel de enterramiento, desafortunadamente removido, acompañado de cerámicas, a veces decoradas mediante incisión o impresión y de una significativa presencia de fauna doméstica (Fernández y Villar 2003); una datación de 4500 \pm 35 BP (GrA-1021) sobre vértebra humana la emplaza en el último tercio del IV milenio AC. Un nivel suprayacente, en este caso sin evidencias funerarias, se data entre los siglos XIX y XIII $\mathrm{AC}$, igualmente con predominio de fauna doméstica y un elenco cerámico dominado por los vasos sin decorar.

Las cavidades de Valdavara y Pala da Vella comparten una localización semejante dentro del macizo montañoso suroriental de Galicia y muestran una coincidencia cronológica en sus dos episodios de ocupación durante la Prehistoria reciente, encuadrables en el Neolítico Final y Bronce Medio, respectivamente. Por otra parte, en ambas se documenta una utilización como lugar de inhumación, en el caso de Pala da Vella limitada a su primera etapa. Si bien las dos tienen grandes similitudes en la composición de los registros arqueológicos de sus niveles superiores (enterramientos secundarios, presencia de colgantes, ajuar cerámico, restos de fauna, etc.) sus respectivas colecciones alfareras muestran, sin embargo, escasas concordancias por lo que a las pautas decorativas se refiere, aún dentro de un encuadre cultural genéricamente tardoneolítico (Valdavara 2 no ha proporcionado esta clase de artefactos) y de la existencia de referentes en otros yacimientos tanto galaicos como de la Meseta septentrional. Estas diferencias entre yacimientos próximos y con ubicación geográfica semejante podrán responder -con la salvedad inherente a la limitada intervención arqueológica en ambos lugares - tal vez a la existencia de tradiciones locales en un medio tan compartimentado como el serrano.

En Valdavara 1 sólo se han recuperado dientes y falanges, elementos de pequeño tamaño que suelen quedar en lugares donde se han recogido los huesos de mayor tamaño para depositarlos en un segundo lugar. La manipulación y 
transporte de los esqueletos humanos es una práctica frecuente documentada en otros yacimientos del Neolítico, Calcolítico y Edad del Bronce de la Península Ibérica (Bueno et al. 2005; Cámara Serrano 2001; Cáceres et al. 2007). Estas prácticas de tipo ritual conllevan la modificación de los depósitos funerarios y el traslado de parte de los restos humanos. De esta forma se pueden caracterizar depósitos primarios o secundarios de huesos humanos a partir de la disposición espacial, existencia de conexión anatómica entre los elementos, frecuencia relativa entre las regiones anatómicas y tamaño de los elementos óseos del conjunto. Por tanto, y a la espera de que la continuación de los trabajos proporcione nuevas evidencias, los restos humanos de Valdavara 1 serían el resultado de inhumaciones primarias de las que posteriormente se habrían extraído los elementos de mayor tamaño para depositarlos en otro lugar.

Por otra parte la documentación de sendos individuos infantiles en el sector 2 de Valdavara nos permitirá aproximarnos, una vez concluya su excavación y estudio, a la esfera funeraria en el Bronce Medio del noroeste, sobre el que no hace una década apenas disponíamos de alguna fecha radiocarbónica y poca más información (Fábregas y Vilaseco 1998). Lo que podemos decir de momento es que en Valdavara 2 se han recuperado huesos del cráneo, huesos largos y costillas pero están ausentes los huesos de mano, huesos de pie y vértebras. Es decir, los elementos de menor tamaño están ausentes y este patrón concuerda con un depósito secundario donde se abandonan los huesos de mayor tamaño que son reconocidos y recogidos más fácilmente (Cámara Serrano 2001; Cáceres et al. 2007). En consecuencia, podría tratarse de un depósito secundario donde se han mezclado los huesos de dos individuos infantiles.

En lo que respecta a la caracterización cronocultural del nivel 4 de Valdavara 1, las primeras fechas obtenidas son especialmente relevantes si tenemos en cuenta la escasez de dataciones y de conjuntos correspondientes al Paleolítico Superior en territorio gallego. Esta escasez constituye una clara anomalía, dada su proximidad a la Cornisa Cantábrica, en la que se documenta la mayor concentración de conjuntos de Paleolítico Superior de toda la Península Ibérica. Dejando a un lado el caso de la Cueva de A Valiña, cuya asignación cronocultural no está exenta de incerti- dumbres, el Paleolítico Superior gallego cuenta con un reducido número de localizaciones, la mayoría situadas al norte de la provincia de Lugo, atribuidas a los momentos finales del Magdaleniense (López Cordeiro 2003; Ramil 1997; Villar Quinteiro 1997): Dos Niñas, Férvedes II, Pena Grande y Prado do Inferno. Según Villar Quinteiro (1997), Férvedes II y Dos Niñas podrían corresponder, en función de sus características tipológicas, a un Magdaleniense Inferior, momento que, a juzgar por las fechas, estaría representado en el nivel 4 de Valdavara 1. Estas localizaciones corresponden en general a abrigos rocosos o a yacimientos al aire libre, en los que hay una deficiente conservación de los restos orgánicos y, por tanto, no ha sido posible obtener dataciones radiométricas.

Los paralelos más próximos a las fechas obtenidas en el nivel 4 de Valdavara 1 se encuentran en conjuntos atribuidos al Magdaleniense Inferior y Medio de la Cornisa Cantábrica, región en la que dichos estadios cronoculturales se encuentran especialmente bien representados. Hay que tener en cuenta que el río Navia, en cuya cuenca se localiza la cueva de Valdavara, constituye una vía natural de penetración desde la costa cantábrica hacia el interior de Galicia y la cuenca del Miño. Desde este punto de vista, no sería de extrañar una vinculación entre el registro arqueológico de la cueva de Valdavara y la secuencia del Paleolítico Superior cantábrico. En concreto, los conjuntos cantábricos que han arrojado fechas similares a las del nivel 4 de Valdavara 1 se enmarcan culturalmente entre los momentos finales del Magdaleniense Inferior y los inicios del Magdaleniense Medio (Corchón 2005; González Sainz y Utrilla 2005).

Las referencias más cercanas se localizan en el sector central y oriental de Asturias, especialmente en las cuencas del Nalón y del Sella: los niveles XII y XI de la cueva de Las Caldas, atribuidos al Magdaleniense Inferior y datados respectivamente en $14495 \pm 140(\mathrm{Ua}-2735)$ y $13755 \pm 120$ BP (Ua-2734), y los niveles IXc-IV del mismo yacimiento, correspondientes al Magdaleniense Medio, y con una serie de fechas comprendidas entre los $13650 \pm 140(\mathrm{Ua}-10190)$ y los $12869 \pm$ 160 BP (Ly-3318) (Corchón 1999); el nivel 3c de la cueva de La Güelga, atribuido a un Magdaleniense Inferior y con tres fechas comprendidas entre los $14170+1030 /-910(\mathrm{GrN}-19387)$ y los $14020 \pm 130$ BP (GrN-17255) (Menéndez et al. 
2005); el nivel B de Entrefoces, asignado al Magdaleniense Inferior y datado en $14690 \pm 200 \mathrm{BP}$ (Ly-2937) (González Morales 1990); el Magdaleniense Medio del nivel 6 de La Paloma, con una fecha de $14600 \pm 160$ BP (OxA-974) (algo antigua para un Magdaleniense Medio); finalmente, también la Cueva de Tito Bustillo ha proporcionado un conjunto de fechas en la línea de las obtenidas en el nivel 4 de Valdavara 1 [nivel 1a: $14250 \pm 300$ (CSIC-154), $14220 \pm 180$ (CSIC-261); nivel 1c: $13870 \pm 220$ (I-8331), $13520 \pm 220$ BP (I-8332)], aunque en este caso hay problemas de incoherencia entre dichas fechas y la sucesión estratigráfica, así como una cierta falta de correspondencia entre algunas de las fechas y la atribución cronocultural que se desprende de las características del conjunto (González Sainz 1989; Moure 1975).

En resumen, los resultados aportados por la primera campaña de excavaciones en la cueva de Valdavara ponen de manifiesto el interés de este yacimiento para el conocimiento de distintos períodos de la Prehistoria gallega. Al mismo tiempo, indican el potencial arqueológico de las formaciones cársticas del este de Galicia como emplazamientos en los que documentar amplias secuencias arqueológicas en las que, además de la profundidad temporal, destaca la buena conservación de restos orgánicos, poco habitual en otros contextos arqueológicos del noroeste peninsular. Esto es especialmente significativo en relación con las evidencias paleolíticas. En cualquier caso, para establecer con precisión la relevancia de este yacimiento $\mathrm{y}$, sobre todo, determinar la presencia de niveles arqueológicos aún más antiguos, habrá que aguardar a los datos que proporcionen las próximas campañas.

\section{AGRADECIMIENTOS}

Las excavaciones en la cueva de Valdavara se realizan gracias al apoyo económico del Concello de Becerreá. Estos trabajos forman parte del proyecto de investigación Ocupaciones humanas durante el Pleistoceno en la cuenca media del Miño (HUM-63662), dirigido por el Prof. Ramón Fábregas Valcarce. También queremos agradecer a Alberto López Pérez, propietario de los terrenos donde se ubica el yacimiento, la autorización para llevar a cabo la intervención, y a Ge- rard Campeny las fotografías correspondientes a las láminas V, VI y VII. Por último, queremos dar las gracias a los dos evaluadores anónimos por sus útiles comentarios.

\section{BIBLIOGRAFÍA}

Bettencourt, A.M.S. (e.p.): "Estruturas e práticas funerárias do Bronze Inicial e Médio do Noroeste Peninsular”. En J. Sánchez Palencia, A. Gilman y P. Bueno (eds.): Libro de homenaje a Maria Dolores Fernández-Posse y de Arnáiz. Bibliotheca Praehistorica Hispana. CSIC. Madrid.

Bueno Ramírez, P.; Barroso, B. y Balbín Behrmann, R. 2005: "Ritual campaniforme, ritual colectivo: la necrópolis de cuevas artificiales del valle de las Higueras, Huecas, Toledo". Trabajos de Prehistoria 62: 67-90.

Cáceres, I.; Lozano, M. y Saladié, P. 2007: "Evidence for bronze age cannibalism in El Mirador Cave (Sierra de Atapuerca, Burgos, Spain)". American Journal of Physical Anthropology 133: 899-917.

Cámara Serrano, J.A. 2001: El Ritual funerario en la prehistoria reciente en el sur de la Península Ibéri$c a$. British Archaelogical Reports, International Series S913. Archaeopress. Oxford.

Corchón, M. ${ }^{\text {aS }}$. 1999: "Solutrense y Magdaleniense del oeste de la cornisa cantábrica: dataciones $14 \mathrm{C}$ (calibradas) y marco cronológico". Zephyrus 52: 3-32.

Corchón, M.aS. 2005: "El Magdaleniense en la Cornisa Cantábrica: nuevas investigaciones y debates actuales". En N.F. Bicho (ed.): O Paleolítico. Actas do IV Congresso de Arqueologia Peninsular (Faro 2004): 15-38. Faro.

Cuenca-Bescós, G.; Straus, L.G.; González, M.R. y García Pimienta, J.C. 2009: "The reconstruction of past environments through small mammals: from the Mousterian to the Bronze Age in El Mirón Cave (Cantabria, Spain". Journal of Archaeological Science 36: 947-955.

Fabián García, J.F. 2006: El IV y III milenio AC en el Valle Amblés (Ávila). Arqueología en Castilla y León, 5. Junta de Castilla y León. Valladolid.

Fábregas Valcarce, R.; Alonso Fernández, S.; Lazuén, T.; Lombera Hermida, A. de; Pérez Alberti, A.; Rodríguez Álvarez, X.P.; Rodríguez Rellán, C.; Terradillos, M.; Serna, M.R. y Vaquero, M. 2008: "Aportacións ó estudo da Prehistoria da cunca media do Miño. Os asentamentos en cova e ó aire libre". Gallaecia 27: 63-88.

Fábregas Valcarce, R.; Bonilla Rodríguez, A. y César Vila, M. 2007: Monte dos Remedios (Moaña. Pon-

T. P., 66, N. ${ }^{\circ}$ 1, enero-junio 2009, pp. 99-113, ISSN: 0082-5638 doi: $10.3989 /$ tp.2009.09014 
tevedra). Un asentamiento de la Prehistoria reciente. Tórculo Edicións. Santiago de Compostela.

Fábregas Valcarce, R. y Fuente Andrés, F. 1988: Aproximaciones a la cultura material del megalitismo gallego: La industria lítica pulimentada y el material cerámico. Arqueohistórica 2. Santiago de Compostela.

Fábregas Valcarce, R.; Lazuén, T.; Lombera, A. de; Peña, J.A.; Pérez Alberti, A.; Rodríguez, X.P.; Rodríguez Rellán, C. y Terradillos, M. 2007: "Novos achados paleolíticos no interior de Galicia. A depresión de Monforte de Lemos e as súas industrias líticas". Gallaecia 26: 7-30.

Fábregas Valcarce, R.; Rodríguez Rellán, C.; Gómez Fernández, A. y Vilaseco Vázquez, X. I. 2005: Prospeccion arqueolóxica intensiva do fenómeno megalítico na Comarca do Deza. Memoria Interpretativa. Servicio de Arqueoloxía da Xunta de Galicia. Santiago de Compostela.

Fábregas Valcarce, R. y Vilaseco Vázquez, I. 1998: "Prácticas funerarias no Bronce do Noroeste". En R. Fábregas (ed.): A Idade do Bronce en Galicia: novas perspectivas. Ediciós do Castro. Sada: 191-219.

Fernández Rodríguez, C. 1993: "Los macromamíferos del Pleistoceno y Holoceno inicial en el Noreste peninsular”. En A. Pérez Alberti, L. Guitián y P. Ramil Rego (eds.): La evolución del paisaje en las Montañas del entorno de los Caminos Jacobeos. Xunta de Galicia. Santiago: 183-191.

Fernández Rodríguez, C. y Villar Quinteiro, R. 2003: "Prospección y excavación de cuevas en la cuenca del Sil (Rubiá, Ourense): La Pala da Vella". Brigantium 14: 13-22.

Fernández Rodríguez, C.; Villar Quinteiro, R.; Llana Rodríguez, C. y Doval Galán, F.J. 1995: "Prospección arqueológica de cavidades en la Sierra de la Encina de la Lastra (Rubiá, Ourense): Primeros resultados". En Actas del XXII Congreso Nacional de Arqueología (Vigo, 1993) II: 43-48. Vigo.

González Morales, M. 1990: "El abrigo de Entrefoces (1980-1983)". Excavaciones arqueológicas en Asturias 1983-86 Oviedo: 29-36.

González Sainz, C. 1989. El Magdaleniense Superior-Final de la región cantábrica. Universidad de Cantabria. Santander.

González Sainz, C. y Utrilla, P. 2005: "Problemas actuales en la organización y datación del Magdaleniense de la Región Cantábrica". En N.F. Bicho (ed.): O Paleolítico. Actas do IV Congresso de Arqueologia Peninsular (Faro, 2004): 39-47. Faro.

Grandal, A. 1991. "Revisión de los fondos paleontológicos del Museo Provincial de Lugo: nuevos datos sobre fauna cuaternaria de Galicia". Cuadernos do Laboratorio Xeolóxico de Laxe 16: 23-35.
Krogman, W.M. e Iscan, M.Y. 1986: The Human Skeleton in Forensic Medicine. Charles C. Thomas. Springfield, IL.

Laplana, C.; Sevilla, P.; López Martínez, N. y Corchón, M.S. 2006: "Primeros datos sobre los micromamíferos (Roedores, Insectivoros, Quirópteros y Lagomorfos) del Solutrense (Pleistoceno Superior final) de la Cueva de Las Caldas (Oviedo, Asturias)”. En E. Fernández Martínez (ed.): Libro de Resúmenes. XXII Jornadas de Paleontología (León, 2006): 137-139. León.

López Cordeiro, M. ${ }^{a}$ M. 2003: "El yacimiento epipaleolítico de Chan da Cruz (Valadouro, Lugo): Síntesis de los primeros resultados". Complutum 14: 39-54.

Menéndez Fernández, M.; García Sánchez, E. y Quesada, J.M. 2005: "Magdaleniense inferior y territorialidad en la Cueva de La Güelga (Asturias)". En N.F. Bicho (ed.): O Paleolitico. Actas do IV Congresso de Arqueologia Peninsular (Faro, 2004): 63-75. Faro.

Moure, J.A. 1975: "Cronología de las industrias tardiglaciares en el Norte de España". Trabajos de Prehistoria, 32: 21-34.

Peman, E. 1990: "Los Micromamíferos de la Cueva de Amalda y su significado. Comentarios sobre Pliomys lenki (Heller, 1930) (Rodentia, Mammalia)". En J. Altuna, A. Baldeón y K. Marezkurrena (eds.): La Cueva de Amalda (Zestoa, País Vasco): Ocupaciones Paleolíticas y Postpaleoliticas. Eusko Ikaskuntza. San Sebastian: 225-238.

Pokines, J.T. 1998: The Paleoecology of Lower Magdalenian Cantabrian Spain. British Archaeological Reports, International Series S713. Oxford.

Ramil, E. 1997: "La transición del Paleolítico Superior al Neolítico en las sierras septentrionales de Galicia. Una aproximación preliminar". En R. de Balbín y P. Bueno (eds): II Congreso de Arqueología Peninsular (Zamora, 1996) I: 273-285. Zamora.

Schener, L. y Black, S. 2000: Developmental Juvenile Osteology. London. Academic Press.

Sevilla, P. 1988: "Estudio Paleontológico de los Quirópteros del Cuaternario Español". Paleontología y Evolución 22: 113-233.

Smith, B.H. 1991: "Standards of human tooth formation and dental age assessment". En M.A. Kelley y C.S. Larsen (eds.): Advances in Dental Anthropology. Wiley-Liss. New York: 143-168.

Ubelaker, D.H. 1989: Human skeletal remains: excavations, analysis, interpretation Taraxacum. Washington D.C.

Vázquez-Monxardín Fernández, A. 1988: “Os materiais arqueolóxicos das covas da Serra da Encina da Lastra". Furada, Revista dos Espeleólogos Galegos 2: 35-40. 
Vera, J.A. (ed.). 2004: Geología de España. Instituto Geológico y Minero de España/Sociedad Geológica de España. Madrid.

Villar Quinteiro, R. 1997: "El Paleolítico Superior y Epipaleolítico en Galicia”. Zephyrus 50: 71-106.
Weninger, B. y Jöris, O. 2004. "Glacial Radiocarbon Age Calibration. The CalPal Program". En T. Higham, C.B. Ramsey y C. Owen (eds.): Radiocarbon and Archaeology. Oxford University School of Archaeology. Oxford: 9-15. 